Supporting Information

\title{
Ultra-fine fiber Raman Probe with High Spatial Resolution and Fluorescence Noise Reduction
}

\author{
Toshiro Yamanaka, ${ }^{+*}$ Hiroe Nakagawa, ${ }^{\dagger}$ Manabu Ochida, ${ }^{\dagger}$ Shigetaka Tsubouchi, ${ }^{\dagger}$ \\ Yasuhiro Domi, ${ }^{\dagger}$ Takayuki Doi, ${ }^{\dagger}$ Takeshi Abe ${ }^{\ddagger^{* *}}$ and Zempachi Ogumi ${ }^{\dagger}$ \\ †Office of Society-Academia Collaboration for Innovation, Kyoto University, Gokasho, Uji, Kyoto 611- \\ ool1, Japan, \\ ${ }^{\ddagger}$ Graduate School of Engineering, Kyoto University, Nishikyo-ku, Kyoto 615-8510, Japan
}

Corresponding author:

*yamanaka@saci.kyoto-u.ac.jp)

**abe@elech.kuic.kyoto-u.ac.jp 

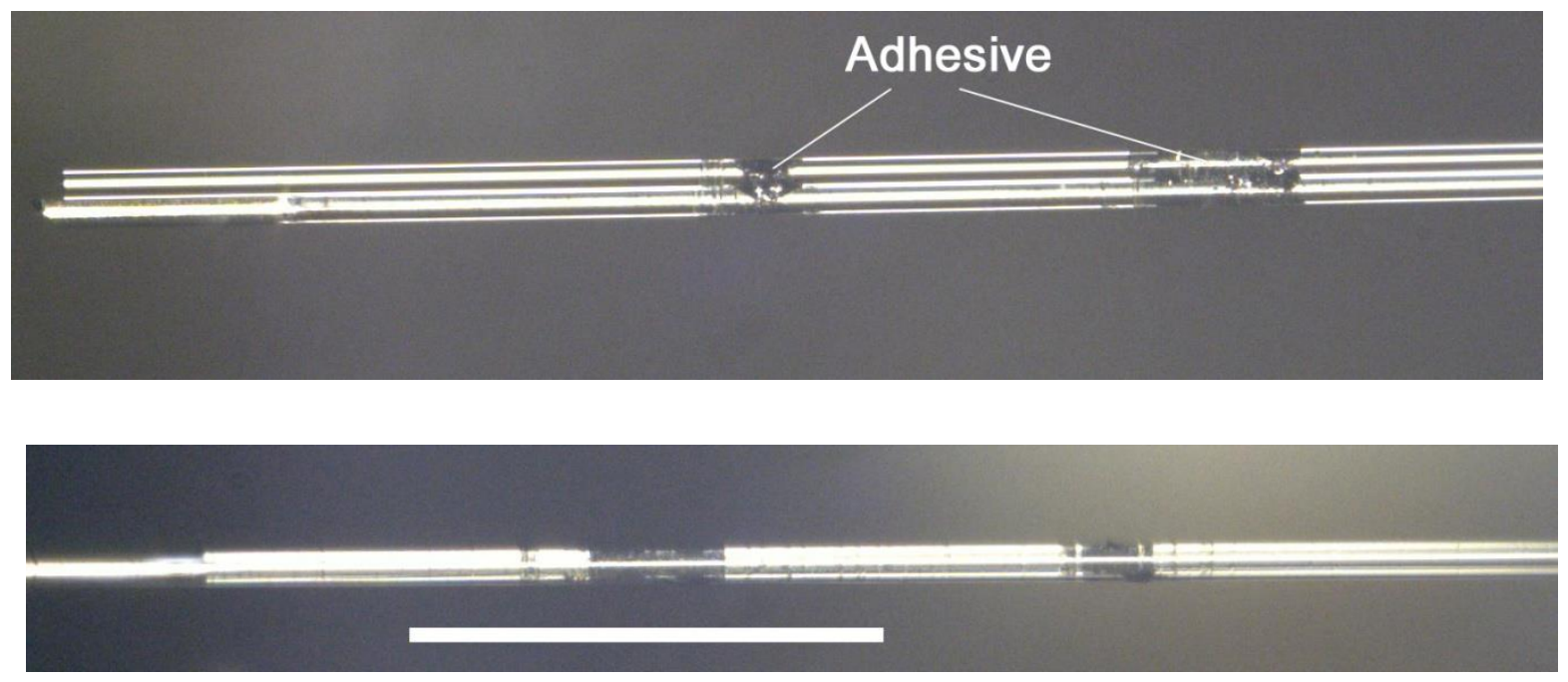

Figure S1. Connection of an excitation fiber and a collection fiber. The two fibers are connected by inorganic adhesive at two points. The width and thickness of the probe are about $60 \mu \mathrm{m}$ and $30 \mu \mathrm{m}$, respectively. The scale bar indicates $300 \mu \mathrm{m}$. 

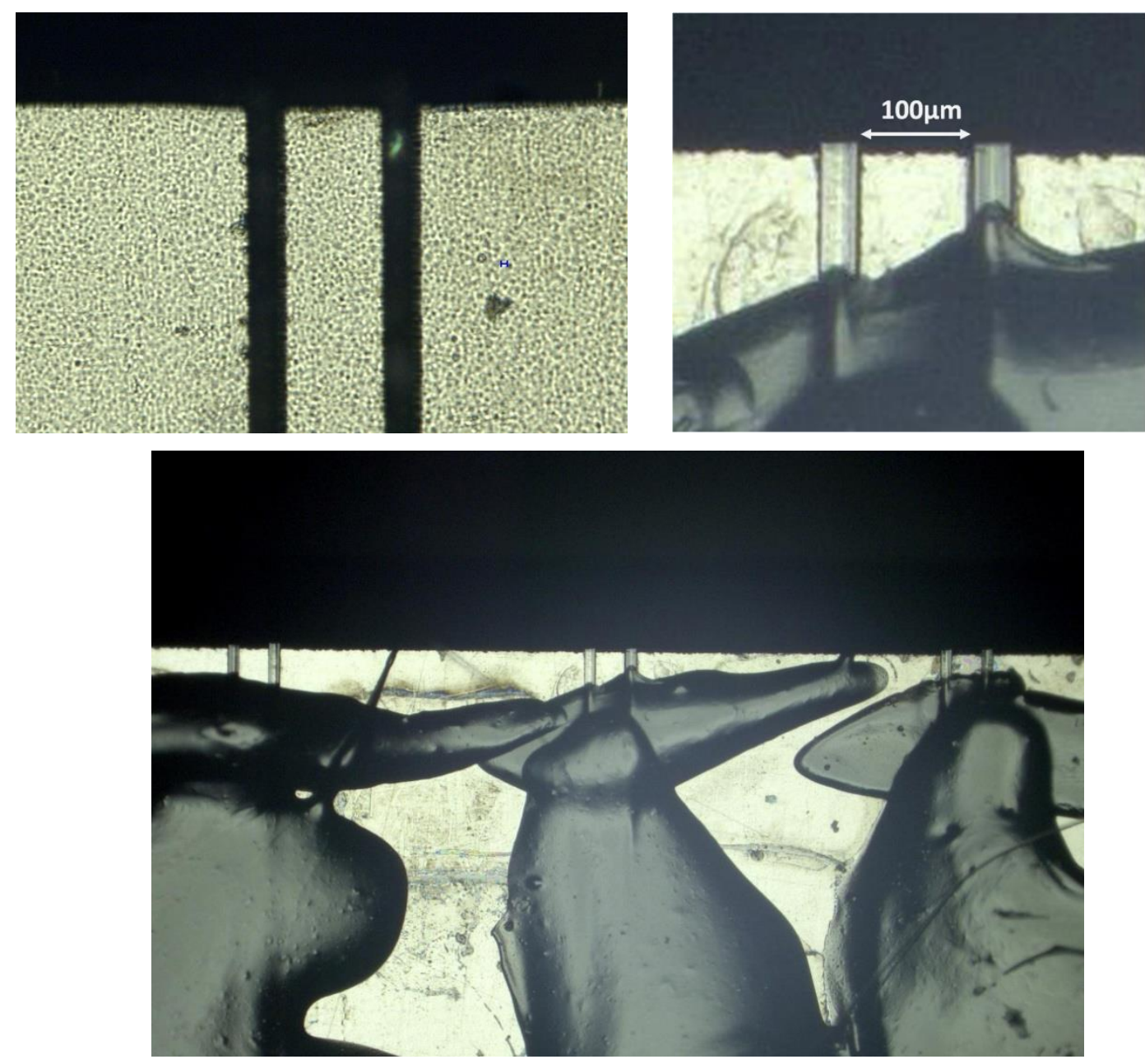

Figure S2. Alignment of the excitation fibers and collection fibers. The two ends of the fibers were aligned by using a glassy carbon block (upper left) with pairs of ditches of $30 \mu \mathrm{m}$ in width. After alignment, the two fibers were fixed using black nail polish. The glassy carbon block had ten pairs of ditches for multiprobe measurements. The lower panel shows mounting of three probes. 


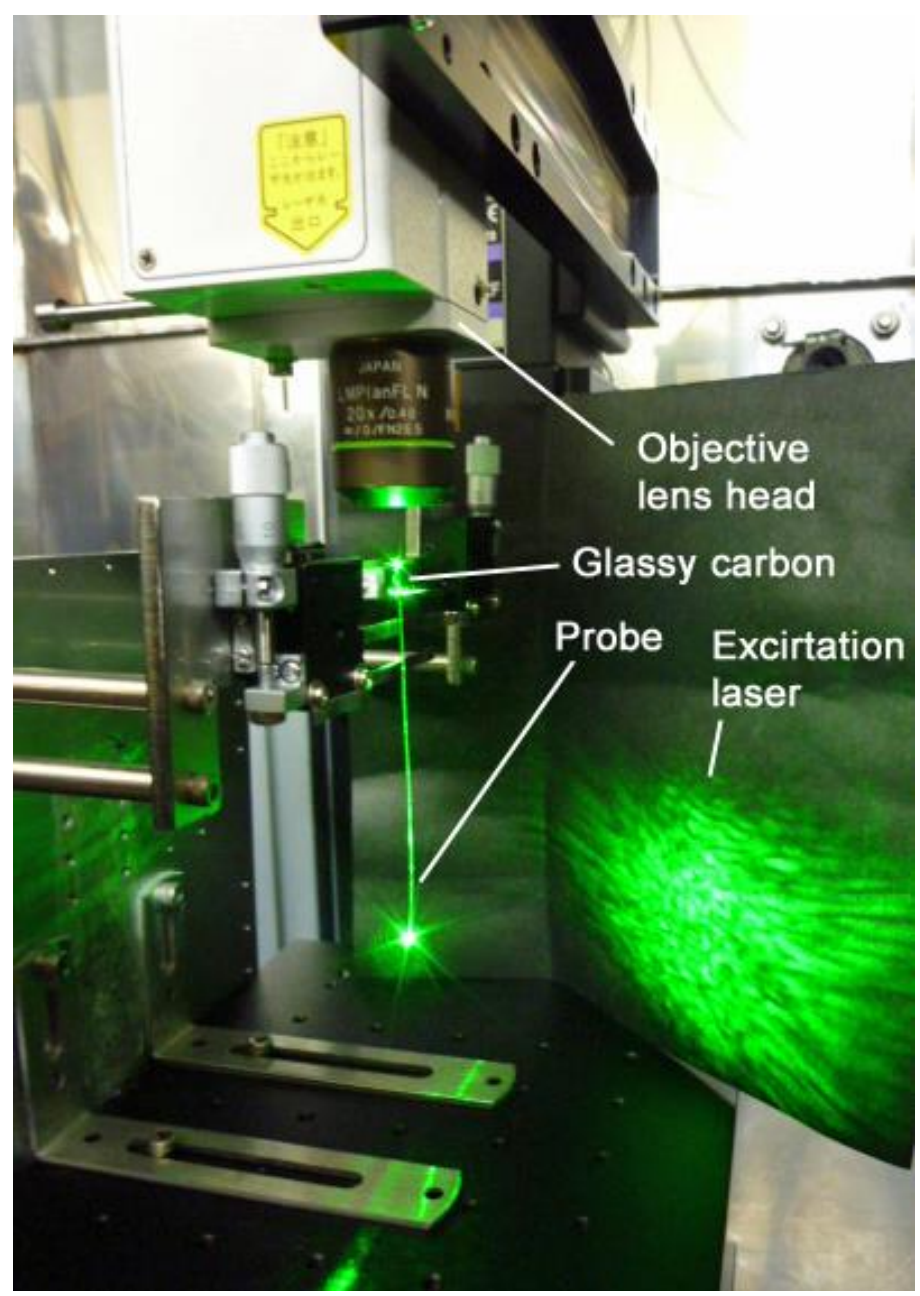

Figure S3. Typical setting for measurements with the probe. The objective lens head was mounted on a movable stage with three stepping motors for movement along $\mathrm{x}, \mathrm{y}$ and $\mathrm{z}$ axes. The excitation laser from the objective lens was focusd on the end of the excitation fiber shown in Fig. S2. For multiprobe measurements shown in Fig. 4 in the main text, three probes were selected by moving the stage by turns. Selection of probes was controlled by a personal computer. 


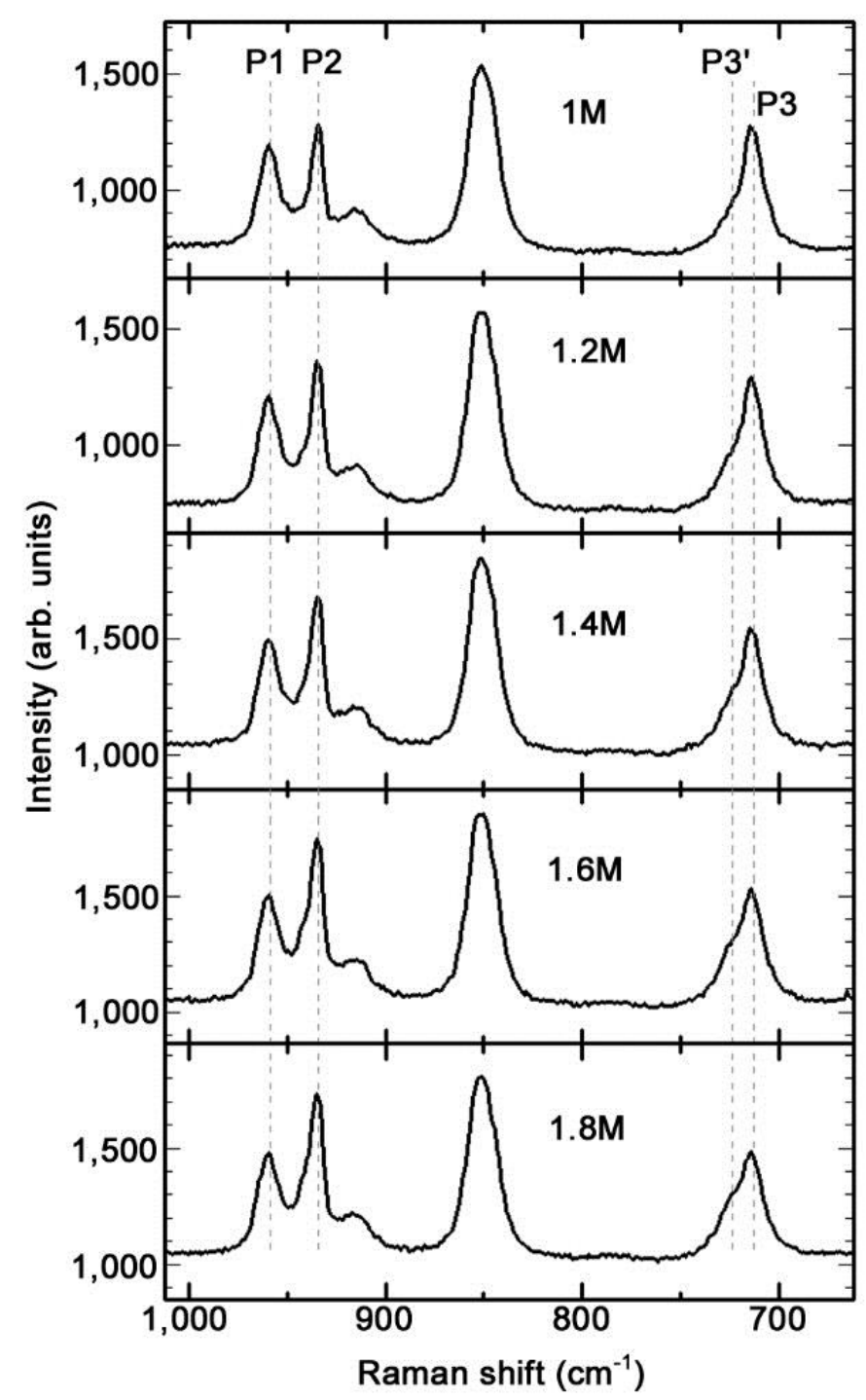

Figure S4. Raman spectra of solutions of $\mathrm{LiClO}_{4}$ dissolved in $\mathrm{PC}$ at concentrations of $\mathrm{LiClO}_{4}$ from $1 \mathrm{M}$ to $1.8 \mathrm{M}$. The spectrum for $1 \mathrm{M}$ agrees well with that in Fig. $3 \mathrm{~d}$ in the main text. The ratio of peak height of $\mathrm{P} 2$ to that of $\mathrm{P} 1$ in the spectrum in Fig. 3e in the main text is about 1.4. The concentration was concluded to be $1.5 \pm 0.2 \mathrm{M}$ by comparing with the spectra for 1.4 $\mathrm{M}$ and $1.6 \mathrm{M}$ in this figure. 


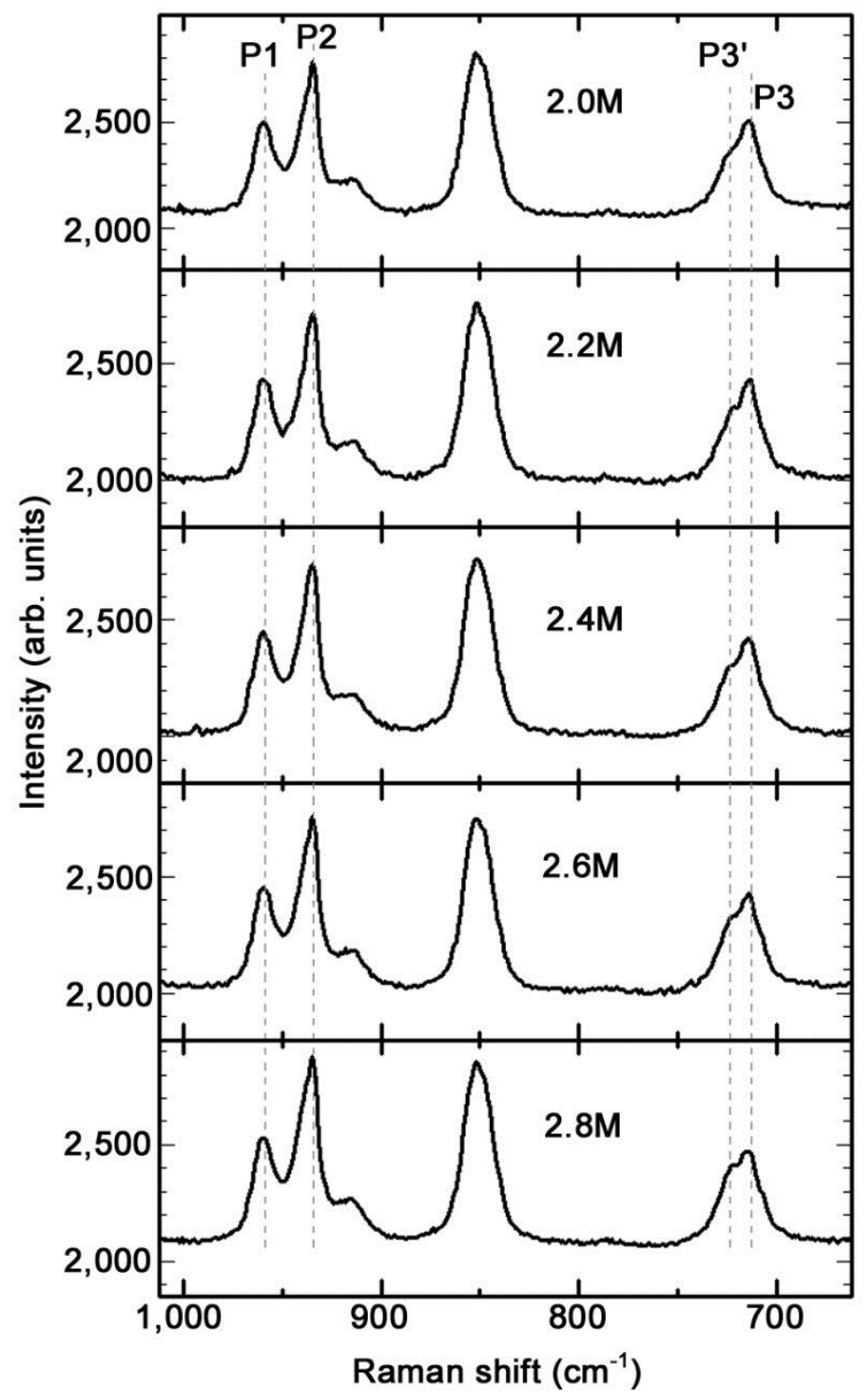

Figure S5. Raman spectra of solutions of $\mathrm{LiClO}_{4}$ dissolved in $\mathrm{PC}$ at concentrations of $\mathrm{LiClO}_{4}$ from $2 \mathrm{M}$ to $2.8 \mathrm{M}$. The ratio of peak height of $\mathrm{P} 2$ to that of $\mathrm{P} 1$ in the spectrum in Fig. $3 \mathrm{f}$ in the main text is about 1.6, and the shoulder P3' is observed. The concentration was concluded to be $2.1 \mathrm{M}$ to $2.7 \mathrm{M}$. 


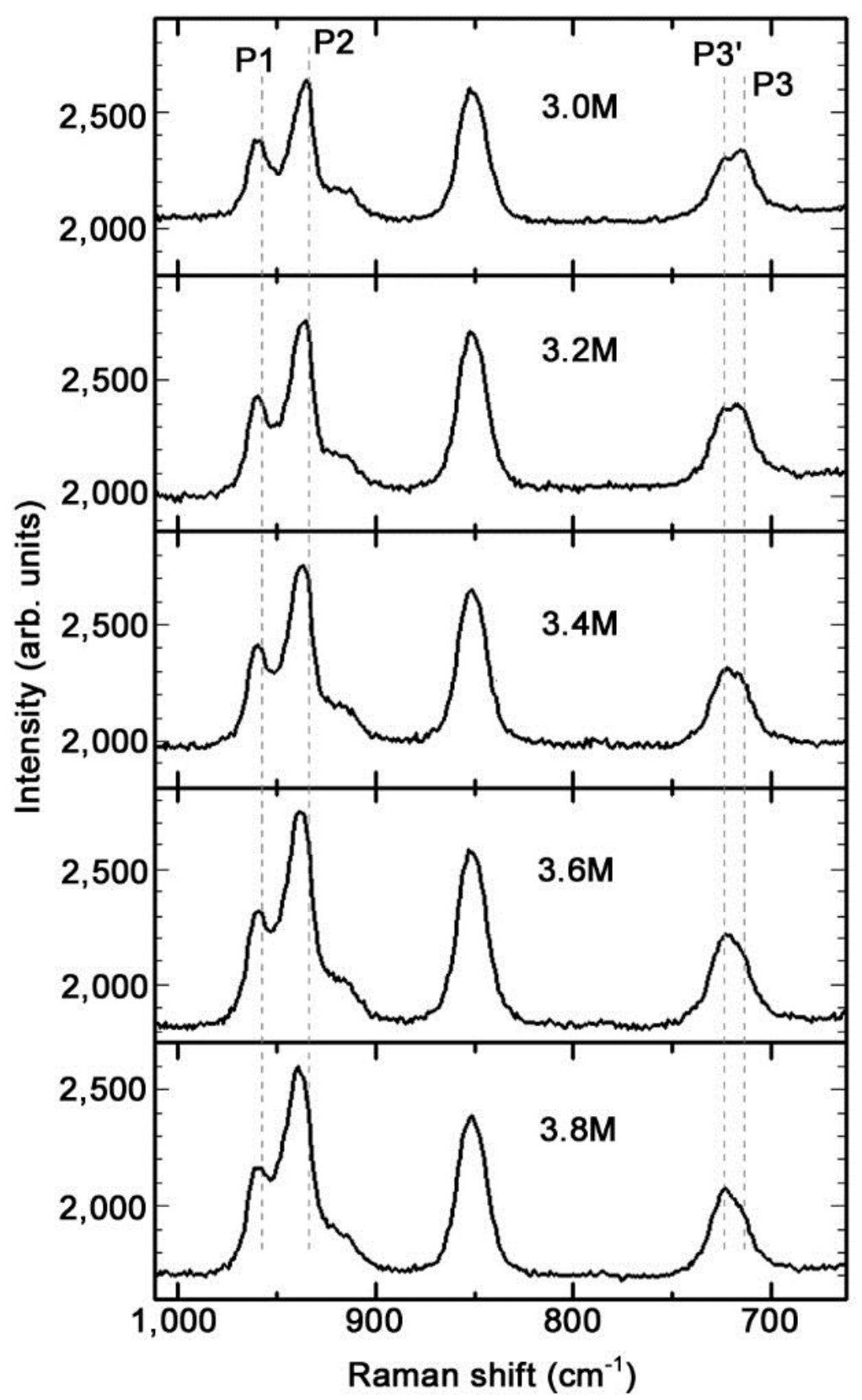

Figure S6. Raman spectra of solutions of $\mathrm{LiClO}_{4}$ dissolved in $\mathrm{PC}$ at concentrations of $\mathrm{LiClO}_{4}$ from $3 \mathrm{M}$ to $3.8 \mathrm{M}$. In Fig. $3 \mathrm{~g}$ in the main text, $\mathrm{P} 3$ ' is dominant over P3. The concentration was concluded to be $3.6 \mathrm{M}$. 


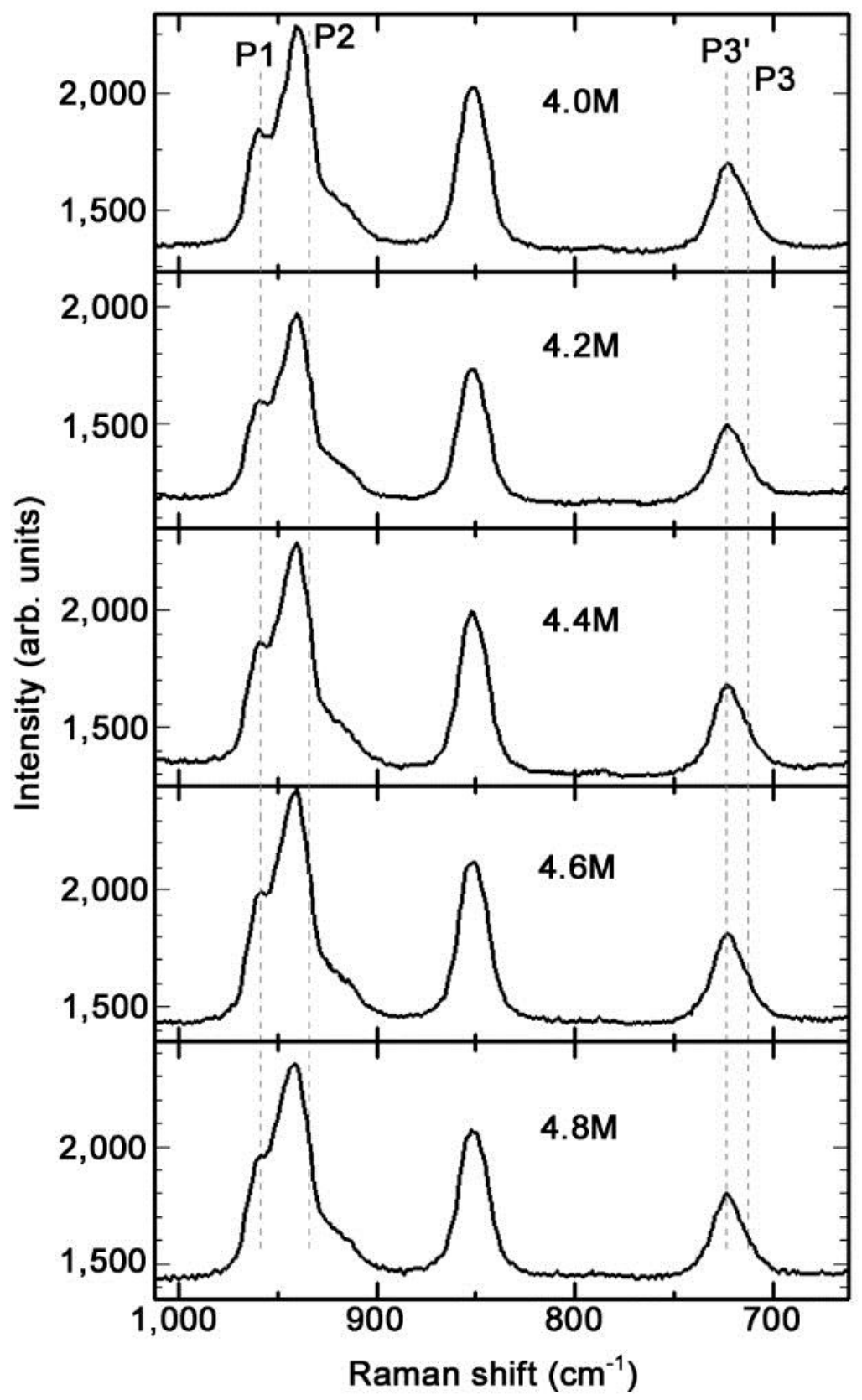

Figure S7. Raman spectra of solutions of $\mathrm{LiClO}_{4}$ dissolved in $\mathrm{PC}$ at concentrations of $\mathrm{LiClO}_{4}$ from $4 \mathrm{M}$ to $4.8 \mathrm{M}$ 

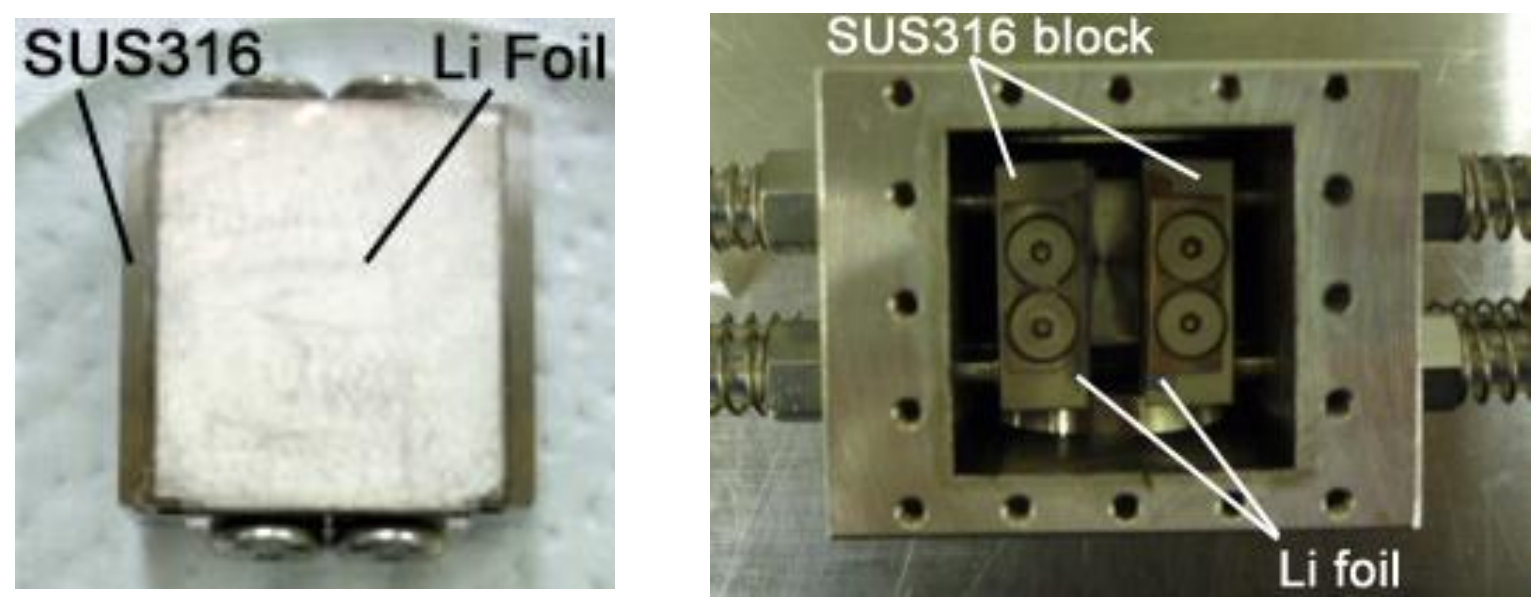

Figure 8. A model electrochemical cell used for analysis in deep narrow spaces by multiprobes. An Li foil was mounted on a very flat surface $( \pm 1 \mu \mathrm{m})$ of an SUS316 block (left). Two Li foils on two blocks were set in a model cell (right). The position of each block was controlled by four micrometers (micromotion mechanisms). The distance between the two flat surfaces of blocks was accurately adjusted by using eight micrometers. 

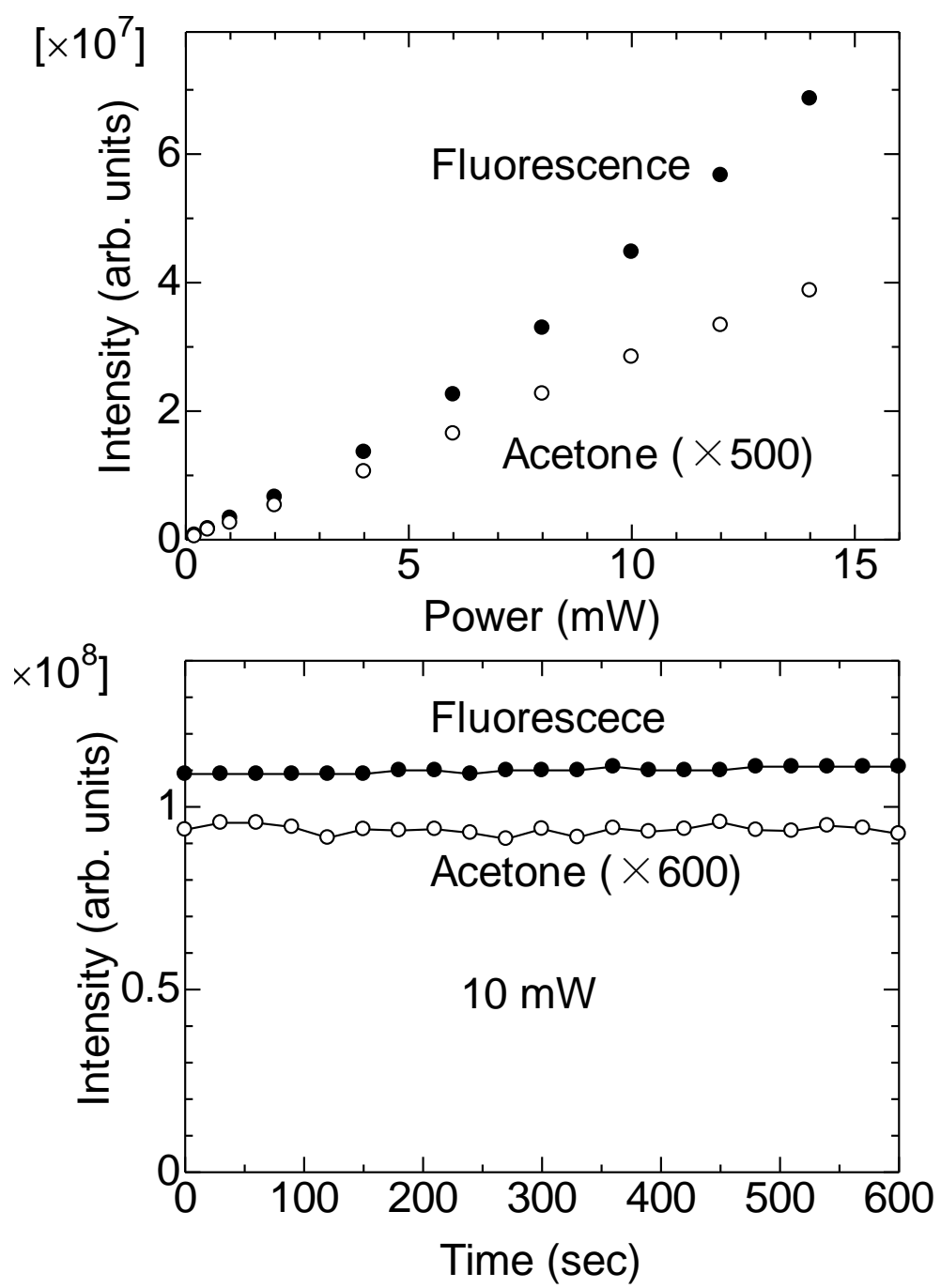

Figure S9. Power dependence and time dependence of intensities of acetone and fluorescence. The liquid sample folder shown in Fig. 6a in the main text was used. The radiuses of the detection area and the focal point of the excitation laser were $10 \mu \mathrm{m}$ and $4 \mu \mathrm{m}$, respectively. $\mathrm{L}$ was set to $0 \mu \mathrm{m}$. The intensities increased almost linearly with the irradiation power. The intensities were almost constant during irradiation for 10 minutes at $10 \mathrm{~mW}$ irradiation power. These results indicate that the irradiation power in the present study is not sufficiently high to induce photobleaching. 


\section{Analysis of a solid surface}

The applicability of the probe to analysis of a solid surface was checked as follows. A polypropylene (PP) film of 15 micrometers in thickness was buried into alumina paste and the resultant block was polished to make a flat surface where a PP band of 15 micrometers in width existed on the alumina surface, as shown in Figs. S10a and S10b. The end of the probe was placed onto the surface (Fig. 10c) and the probe was scanned on the surface in the direction perpendicular to the PP band ( $\delta$ axis). In this scan, the alumina block was actually moved while the probe was fixed as shown in Fig. S10a. $\delta$ was defined to be zero when the center of the PP band was placed at about the center of the analysis area $((Y, Z)=(15,15)$ in Fig. 2e in the main text) as shown in Fig. S10c. The peak area of PP (Fig. 10d) is plotted against $\delta$ in Fig. S10e. The area had a maximum at around $\delta=0$, and the value for FWHM was estimated to be 24 micrometers, which was close to the 23 micrometers estimated from Figs. $2 \mathrm{f}$ and $2 \mathrm{~g}$ in the main text. 

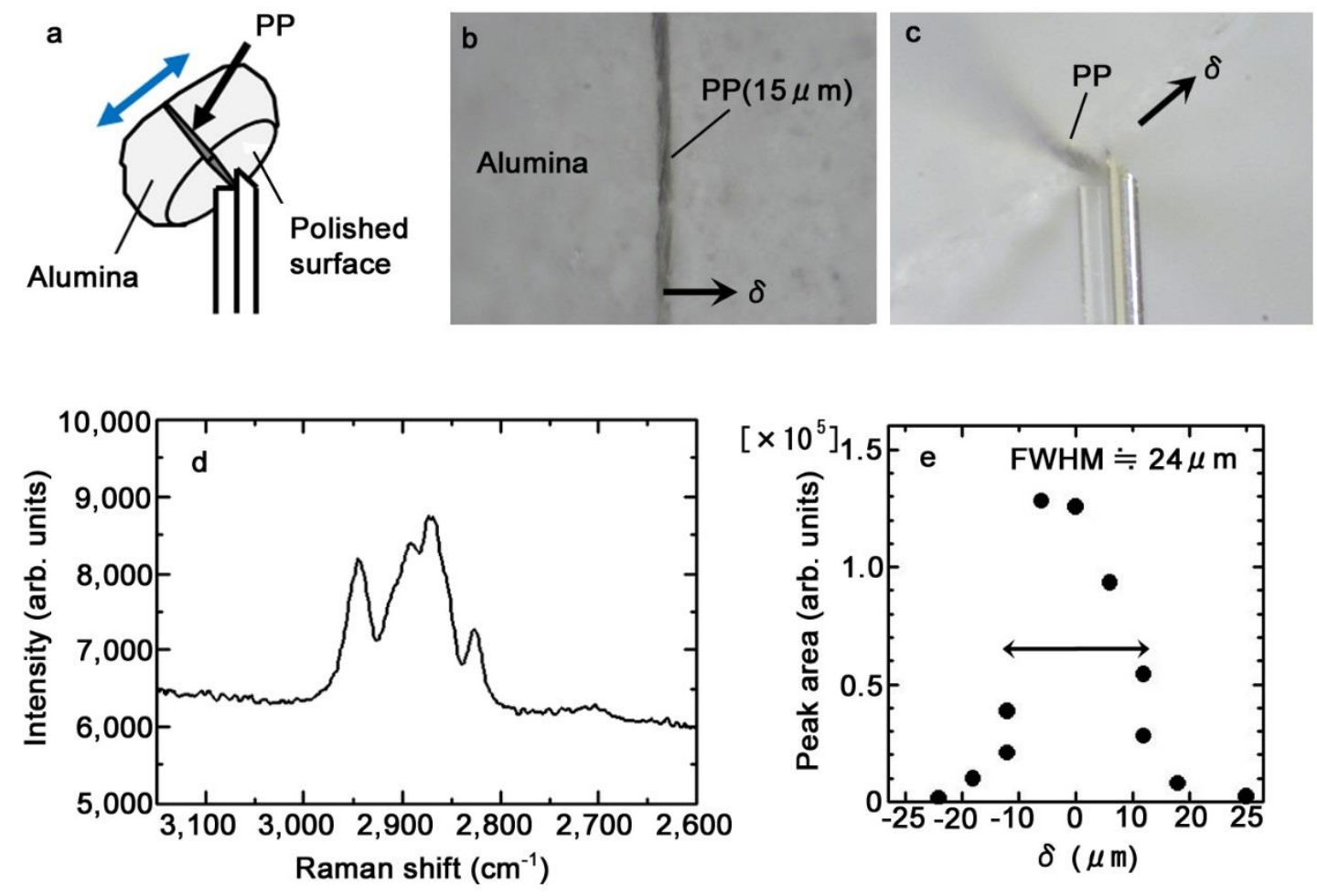

Figure S10. Analysis of a solid surface. (a) Schematic illustration of the method for analysis of an alumina/PP/alumina surface. (b) Photograph of a polished surface of a block of alumina/PP/alumina. A polypropylene (PP) film of 15 micrometers in thickness was buried into alumina paste and the resultant block was polished to make a flat surface with a PP band of 15 micrometers in width existing on the alumina surface. (c) Microscopic observation to confirm the position of the probe relative to the PP band, $\delta . \quad \delta$ is defined to be zero in this picture. (d) Raman spectrum of PP taken at $\delta=0$ (20 s $\times 2$ times). (e) Peak areas of PP plotted against $\delta$. The area had a maximum at around $\delta=0$, and the value for FWHM was estimated to be 24 micrometers. 